\title{
Circadian ingestion behaviors: animal models outlooks
}

\begin{abstract}
This article discusses selected most important factors regulating circadian food ingestion patterns in animal models. Such knowledge is crucial for development of innovative pragmatic perspectives to control and optimize circadian patterns of eating in the modern human exposed to a variety of metabolic disorders on top of which is the growing obesity.
\end{abstract}

Keywords: ingestion, eating behavior, circadian rhythm
Special Issue - 2015

Akbar Nikkhah
Department of Animal Sciences, University of Zanjan, Iran

Correspondence: Akbar Nikkhah, Chief Highly Distinguished Professor, Department of Animal Sciences, Faculty of

Agricultural Sciences, University of Zanjan, Foremost Principal Highly Distinguished Elite-Generating Scientist, National Elite Foundation, Iran, Email anikkha@yahoo.com

Received: November 9,2015 | Published: November 16, 2015

\section{Introduction}

Grazing occurs mainly around sunrise and sunset. In grazing sheep, the maximum jaw movement occurs in the late afternoon before sunset. Dairy cows exhibit three main grazing bouts of dawn, afternoon, and dusk. However, the diurnal patterns in grazing appear to vary across seasons. In Western Australia with hot summers and mild winters, the main summer grazing activity occurs during early morning and late afternoon with a smaller activity around midnight. Sheep and cattle likely have comparable grazing patterns in summer. In winter, however, the cattle show intensive grazing activities not only in the morning and afternoon but also during the period from 2000-0100h. Unlike in summer, in winter, sheep has a progressive grazing activity from 0600 through 1700 with low activity in the evening. Under intensive feeding systems, dairy cows and finishing bulls fed only once daily in the morning exhibit two peaks in eating activity. The greatest one is right after feeding and another around dusk. The eating/grazing pattern has circadian patterns. ${ }^{1}$

Cows seem to stop the morning eating before reaching the maximum rumen capacity. The observation that rumen capacity peaks at dusk but not during the morning and afternoon grazing has implications. It is found that time spent eating during the dusk grazing bout is longer than that during the dawn and afternoon bouts. Moreover, during dawn and afternoon bouts, the cows finish eating before reaching a rumen capacity which was reached during the dusk bout. Therefore, the rumen fill has probably a more significant role in regulating the dusk feed intake compared to dawn and afternoon feed intakes. ${ }^{2,3}$

Despite feeding adequate coarse corn silage as $45 \%$ of diet dry matter (DM), finishing bulls select the coarse particles for as long as $16 \mathrm{~h}$ post-feeding. Such a late selection of the more structured total mixed ration particles is an effort to optimize the intake of long roughage particles. In beef cattle, the herbage allocation time (e.g., $0700 \mathrm{~h}$ vs. $1500 \mathrm{~h}$ ) alters daily patterns in eating, ruminating, and idling behaviors in heifers. The heifers turned unto the ungrazed strip at $0700 \mathrm{~h}$ have more intense evening grazing with faster bite rates compared with the heifers allotted the herbage at $1500 \mathrm{~h}$. The morning grazing is $36-39 \%$ of total daily grazing time in the latter group but only $25-28 \%$ in the former group. The nutritional status of the animal before feeding can affect the post-feeding eating intensity. Fasting, for instance, hastens eating by increasing the bite rate and enlarging the bite mass. However, the data on the effects of feeding strategies on diurnal patterns in feed intake of non-grazing ruminants is scarce..$^{3-5}$

The large evening meals in grazing cows are partly linked to an optimal foraging strategy. The plant content of DM and water soluble carbohydrates rises and NDF decreases as day progresses. The accumulation of highly-digestible nutrients in the evening is due to the daylight photosynthesis in plant leaves. This may partly explain why cattle, sheep, and goats prefer the fresh forage grass and legumes harvested at sunset compared to that harvested at sunrise. A higher in vitro true DM digestibility for PM-cut than for AM-cut alfalfa hay is known. Ruminants may have greater feed intake and greater total tract DM digestibility when they are offered the sunset-cut alfalfa hay instead of the sunrise-cut alfalfa hay. The grazing ruminants appear to learn the time of maximum plant nutrient quality, leading to a more intensive eating in the evening than in the morning. ${ }^{4-6}$

The lower ambient temperature at night vs. day may also contribute to the larger evening meals in grazing ruminants. Additionally, ruminants attempt to optimize their nutrient intake profile by filling the rumen in the evening because usually little grazing occurs overnight. In so doing, microbial growth in the rumen would sustain by the time the morning meal would start. Ruminants have evolved adaptive patterns in ingestion, rumination, and digestion behaviors. Grazing has evolved to occur mostly during the day, particularly around sunset and sunrise, whereas rumination occurs mostly at resting time or overnight. Therefore, despite being incorporated into the intensive production systems, ruminants are still responsive to the signals induced by the environmental synchronizers such as light turn on and off as well as feed quality and availability. $1,4,6$

The eating rate of both concentrate and hay in ad libitum-fed goats is higher during the dark period than during the light period. Also, goats prefer concentrate during dark rather than the light period. Thus, factors regulating feed intake may be of different type and magnitude between dark and light periods. Likewise, a nocturnal appetite for concentrate is known. The meal size is linked to water intake more 
around the light-phase meals than the dark-phase meals. This shows that the factors affecting feed and water intake are of unequal type and magnitude between day and night. When cows are fed once daily a diet composed of hay, corn silage, and grass silage in the morning, regardless of the breed, all cows exhibit diurnal patterns in their eating activity with the maximum activity occurring between $0800-1300 \mathrm{~h}$. Cows also show a night-time preference for the high-energy portion of the diet or grass silage. It seems that during the night ruminants prefer energy dense food. Cows prefer to eat more of the concentrate overnight. These findings imply that short-term, post-meal regulation of feed intake differ between day and night. As day progresses towards night, the cow faces a more negative nutrient balance, and thus, attempts to consume the highest energy in the shortest time. ${ }^{2,3,5}$

The light-dark cycle is an important part of the animal environment. In pigs, feed ingestion peaks around the light turn-on and turn-off. Turning lights on and off seems to act as an inducer, thus stimulating eating activity. This implies that animals can anticipate the time around which the lights are turned on and off. Under fluctuating ambient temperatures, eating rate is the highest around the times when lights are switched on and off. When the ambient temperature is kept constant over the $24 \mathrm{~h}$ period, the pigs eat most intensely within $2 \mathrm{~h}$ after light-on and $3 \mathrm{~h}$ before light-off. This shows that diurnal variations in ambient temperature affect light-mediated feed intake. The fact that ruminants eat and graze most intensively around sunrise and sunset indicates that light intensity or wavelengths are important in stimulating feed intake. The intense evening eating may be an interactive response to several factors rather than being solely related to light intensity. The anticipation of a non-eating or resting phase (overnight) could motivate the animal to fill the rumen to avoid hunger later during the night. Moreover, the more favorable environment reflected in higher herbage quality in the evening than in morning, feed preference, and social facilitation contribute to the intensive evening eating in grazing ruminants. The interaction between light intensity and the herbage quality may induce grazing beyond that achievable during the morning. As a result, the maximum rumen fill may not be achieved during the day. ${ }^{5,6}$ This emphasizes the notion that rumen fill is only one of a multitude of factors regulating feed intake. Diurnal patterns in rumen digesta kinetics are still largely unexplored in non-grazing ruminants, particularly in response to feed delivery time, requiring future research. Animal models findings could be greatly helpful in making new discoveries in human nutrition where great sufferings occur due to suboptimal eating behavior.

\section{Acknowledgments}

Thanks to the Ministry of Science Research and Technology, and National Elite Foundation for supporting the author's global initiatives and programs of optimizing science edification in the third millennium.

\section{Conflict of interest}

Author declares that there is no conflict of interest.

\section{References}

1. Nikkhah A. New Theories of Ruminant Feed Intake Regulation (In Persian). Tehran \& Zanjan: Jahade-Daneshgahi Publishers; 2014.

2. Nikkhah A. Time of feeding an evolutionary science. Germany: LAP LAMBERT Publishing, GmbH \& Co. KG; 2011.

3. Nikkhah A. Father Nutrition. J Nutr Health Food Eng. 2015;2(6):00080.

4. Nikkhah A. Eating time: an evolutionary manager of postmodern rumen physiology and health: a review. Open Access Animal Physiology. 2011;3:13-19.

5. Nikkhah A. Chronophysiology of ruminant feeding behavior and metabolism: an evolutionary review. Biol Rhythm Res. 2013;44(2):197-218

6. Nikkhah A. Animal Chronophysiological Management: An Emerging Bioscience. Open Access Animal Physiology. 2011;3:9-12. 